\title{
AVL9 is Upregulated in and Could Be a Predictive Biomarker for Colorectal Cancer
}

This article was published in the following Dove Press journal:

Cancer Management and Research

\author{
Danfeng $\mathrm{Li}^{1, *}$ \\ Yongming Zeng $\mathbb{D}^{1, *}$ \\ Peilin Shen (D) $^{2, *}$ \\ Xiaosheng Lin' \\ Tian Yang' \\ Binlie Chen ${ }^{1,3}$ \\ Zhiyan $\mathrm{Ma} \mathbb{D}^{1,3}$ \\ Huaiming Wang (D) \\ 'Department of Gastrointestinal Surgery, \\ The First Affiliated Hospital of Shantou \\ University Medical College, Shantou, \\ 5I504I, People's Republic of China; \\ ${ }^{2}$ Department of Urology, The First \\ Affiliated Hospital of Shantou University \\ Medical College, Shantou, 5I504I, \\ People's Republic of China; ${ }^{3}$ Medical \\ College, Shantou University, Shantou, \\ Guangdong, People's Republic of China
}

*These authors contributed equally to this work
Correspondence: Huaiming Wang Department of Gastrointestinal Surgery, The First Affiliated Hospital of Shantou University Medical College Email13750417745@I63.com
Purpose: This study aimed to explore the function and clinical significance of $A V L 9$ in colorectal cancer (CRC).

Materials and Methods: The GEO, TCGA, and GEPIA databases were searched to evaluate the expression level of $A V L 9$, while the SurvExpress online tool was used to explore its related clinical survival prognosis. The cBioPortal and LinkedOmics databases were used to identify $A V L 9$ expression-related genes. Protein-protein interaction (PPI) networks were analyzed using Cytoscape 3.7.1 and DAVID6.8, which was used to perform Gene Ontology (GO) and Kyoto Encyclopedia of Genes and Genome (KEGG) signal pathway enrichment. The immunohistochemistry of $A V L 9$ in CRC was detected using an online tool protein atlas. RNA isolation and reverse transcription-quantitative polymerase chain reaction (RT-qPCR) assays were used to detect $A V L 9$ expression in tissue and plasma samples.

Results: Our study confirmed that $A V L 9$ was highly expressed in CRC lesions versus the adjacent normal tissues $(\mathrm{P}<0.001)$. High $A V L 9$ expression was negatively associated with survival outcomes $(\mathrm{P}<0.05)$. GO analysis showed that $A V L 9$ expression-related genes were enriched in single organismal cell-cell adhesion, post-transcriptional regulation of gene expression, and negative regulation of the vascular endothelial growth factor receptor signaling pathway $(\mathrm{P}<0.05)$. On a KEGG pathway analysis, these genes were mainly involved in progesterone-mediated oocyte maturation, axon guidance, the insulin signaling pathway, and the ubiquitin-mediated proteolysis signaling pathways $(\mathrm{P}<0.05)$. In the PPI analysis, the KBTBD2, KIAA1147, EPDR1, and RNF216 genes interacted with AVL9, and GEPIA predicted that their expression levels were all positively correlated with $A V L 9$. Furthermore, a clinicopathological parameter analysis found that high $A V L 9$ expression was positively correlated with differentiation and TNM stage. RT-qPCR analysis further showed that plasma AVL9 expression was upregulated in CRC patients versus healthy controls.

Conclusion: AVL9 could serve as a potential biomarker and therapeutic target for CRC.

Keywords: AVL9, biomarker, colorectal cancer, prognosis

\section{Introduction}

Colorectal cancer (CRC) is a common malignant tumor worldwide. ${ }^{1}$ According to the latest statistics from the American Cancer Society, CRC is the second most common cause of cancer death in the United States. In the past 10 years, there has been a rapid decline in its incidence among those aged 65 years and older, with a downward trend of approximately $3.3 \%$ annually. However, the incidence in the age group of 50-64 years has increased by 1\% each year, while the incidence of CRC patients under 50 years of age has increased by approximately $2 \%$ annually. The mortality rate showed the same trend in the above age groups. It is worth noting that the mortality rate of $\mathrm{CRC}$ in those age $<50$ years increased by $1.3 \%$ 
per year. ${ }^{2}$ In fact, $90 \%$ of CRC-related deaths can be prevented if patients are diagnosed at an early stage; therefore, early diagnosis is the fundamental way to improve patient prognosis. ${ }^{3}$ The basic reason for poor patient prognosis is that the understanding of the exact molecular mechanism of the early occurrence and development of CRC remains very limited. Therefore, a deeper understanding of the specific pathogenesis of CRC and clarification of the reasons for the increase in morbidity and mortality in young and middle-aged adults can improve the overall prognosis of CRC.

The migration-associated gene AVL9 is located on the human chromosome 7.14:32,495,489-32,588,726 (111,980 nt in length). Recent studies have shown that AVL9 plays an important role in cell polarity, cell migration, and cell cycle progression and acts as an oncogene. ${ }^{4,5}$ Zhang et al revealed that AVL9 expression is upregulated in clear cell renal carcinomas and promotes cell migration. ${ }^{6}$ Moreover, our previous study identified that AVL9 is a target gene of the linc00662/ miR-497-5p axis that promotes the development of CRC. ${ }^{7}$ However, the function and clinical application value of $A V L 9$ in CRC remain largely unexplored, and in the present study, we aimed to explore the roles of AVL9 in CRC and its potential relationship with clinicopathologic parameters and identify whether it may serve as a new target for the diagnosis and treatment of CRC.

\section{Materials and Methods \\ Data Acquisition}

The expression level of $A V L 9$ in CRC was analyzed using GEPIA (http://gepia.cancer-pku.cn/), Gene Expression Omnibus (GEO) (http://www.ncbi.nlm.nih.gov/geo), and The Cancer Genome Atlas (TCGA) databases (https:// www.cancer.gov/about-nci/organization/ccg/research/struc tural-genomics/tcga) databases. Overall survival (OS) and disease-free survival (DFS) analyses were performed using the online database SurvExpress (http://bioinformatica. mty.itesm.mx:8080/Biomatec/SurvivaX.jsp). The online tool protein atlas (https://www.proteinatlas.org/) was used to detect $A V L 9$ expression in different human tumors.

\section{Bioinformatics Analysis}

AVL9 expression-related genes were obtained from LinkedOmics (http://www.linkedomics.org/login.php) and cBioPortal (http://www.cbioportal.org/). A Spearman correlation coefficient of $>|0.5|$ was defined as the screening condition, while a Venn diagram was generated using the online webtool (http://bioinformatics.psb.ugent.be/webtools/Venn/) to identify overlapping related genes based on the screening conditions. Gene Ontology (GO) and Kyoto Encyclopedia of Genes and Genomes (KEGG) signal pathway enrichment analyses of $A V L 9$ expression-related genes were performed using the online biological information database DAVID6.8 (http://david.ncifcrf.gov/) $\quad(\mathrm{P}<0.05)$. The protein-protein interaction (PPI) network was predicted using the STRING database (http://string-db.org), "Multiple proteins" was selected to enter expression-related genes and $A V L 9$, "Homo sapiens" was selected for the species, and "Medium confidence (0.400)" was selected for the confidence of the strength of the interactions between proteins. Then, the networking results were exported in the "TSV" format and imported into Cytoscape 3.7.1.

\section{Tissue Samples, Blood Samples, and Clinical Data Collection}

Human CRC tissues and their paired adjacent noncancerous specimens were collected from 50 patients who had undergone CRC surgical resection without receiving any other treatment before surgery and were pathologically diagnosed with CRC between 2014 and 2016. After excision, the tissues were quickly frozen and stored at $-80^{\circ} \mathrm{C}$. Patients were monitored once every 3 months in the first 2 years after surgery and then every 6 months thereafter; at each followup, a detailed history was collected and a comprehensive physical examination performed; data of routine blood parameters, liver function, plasma carbohydrate antigen19-9, carcinoembryonic antigen, and abdominal ultrasound were all required. OS was calculated from the date of diagnosis until the date of death or the end of the follow-up period. Plasma samples of CRC patients and healthy controls were obtained from The First Affiliated Hospital of Shantou University Medical College. The Ethics Committee of Shantou University Medical College approved this study, and each participant and healthy controls signed an informed consent form regarding their specimen use for scientific research, and the research was performed in accordance with the World Medical Association Declaration of Helsinki.

\section{Quantitative Real-Time Polymerase Chain Reaction}

RNAiso Plus reagent (TaKaRa, Dalian, China) was used to extract total RNA from frozen tissues and plasma samples. According to the manufacturer's instructions, $500 \mathrm{ng}$ of total RNA was reverse transcribed to cDNA using the 
PrimeScript RT Master Mix (TaKaRa). The SYBR Premix Ex Taq II Kit (TaKaRa) was used to determine the expression level of $A V L 9$ using the StepOnePlus system (Applied Biosystems, CA, USA). Fold changes were calculated using the $2-\Delta \Delta \mathrm{Ct}$ method. The primer sequences for $A V L 9$ were as follows: forward 5'-GTGAGGCACGTGA CTGAGAA-3'; and reverse: 5'-TTGTTGCTGTTCCAC ACCCT-3'. The primer sequences for GAPDH were as follows: forward 5'-CGCTCTCTGCTCCTCCTGTTC-3'; reverse: 5'-ATCCGTTGACTCCGACCTTCAC-3'. Data were obtained from at least three independent experiments and are presented as mean \pm standard deviation.

\section{Statistics}

All data are presented as mean \pm standard deviation. The chi-square test was used to analyze the data. Statistical analyses were performed using IBM SPSS 24.0 (IBM, USA). An unpaired $t$-test was used to evaluate the differential expression of $A V L 9$ between cancer tissues and adjacent normal tissues. The Log rank test and Cox proportional hazards regression were used to estimate the prognostic value of $A V L 9$. Receiver operating characteristic (ROC) curve analysis was performed using the "pROC" package ${ }^{8}$ in R software (version 4.03), and significant differences were considered at values of $\mathrm{P}<0.05$.

\section{Results}

\section{Upregulation of AVL9 Predicted Poor Prognosis in CRC Patients}

Based on our previous study and other published research, we first found that the relative expression level of AVL9 was significantly upregulated in CRC tissues in the GEPIA database. The data also indicated that $A V L 9$ expression was upregulated in CRC versus normal tissues in the GEO microarray dataset GSE32323 and dramatically increased in CRC, findings that were consistent with the GEO and GEPIA data (Figure 1A-C). The online tool protein atlas was used to detect the expression of $A V L 9$ in different human tumors, and the results showed that both $A V L 9$ mRNA and protein were significantly expressed in CRC (Figure S1). Immunohistochemical analysis was performed to detect the AVL9 protein expression in 10 CRC tissues, and the results showed that 9 of 10 showed moderate staining and one of 10 showed strong staining. In the intensity analysis, we found that 2 of 10 were strong, while 8 of 10 were moderate. In the quantity analysis, we found that 7 of 10 were more than $75 \%, 2$ of 10 were between $25 \%$ and $75 \%$, and 1 of 10 was less than $25 \%$; therefore, we can conclude that the AVL9 protein is also highly expressed in CRC (Figure S2). To further explore the relationship between AVL9 expression and survival outcomes, KaplanMeier curve analysis using the SurvExpress online tool showed that high expression of $A V L 9$ was significantly associated with poor prognosis in terms of OS (Figure 2A, $\mathrm{P}=0.0433$; Figure $2 \mathrm{~B}, \mathrm{P}=0.01585$ ) and $\mathrm{DFS}$ (Figure $2 \mathrm{C}$, $\mathrm{P}=0.0002$; Figure 2D, $\mathrm{P}=0.0075$ ).

\section{Bioinformatics Analysis of AVL9 and the Genes Associated with Its Expression}

To investigate the potential functions of $A V L 9$ in CRC, the LinkedOmics and cBioportal online bioinformatics databases were used to analyze the $A V L 9$ co-expression genes in CRC in TCGA dataset, we generated a Venn diagram using an online web tool (http://bioinformatics.psb.ugent.
A

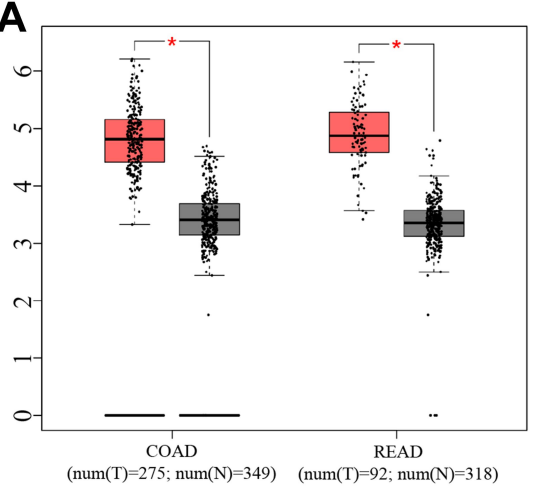

B

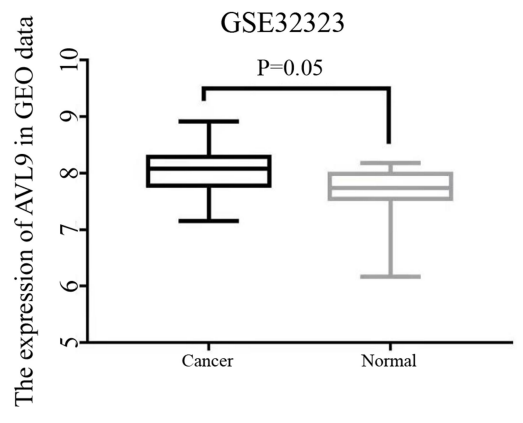

C

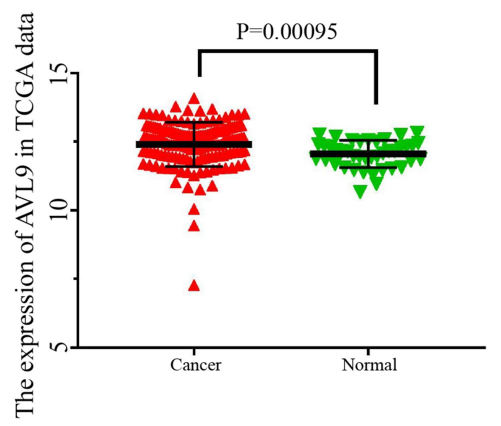

Figure I AVL9 expression in CRC and normal tissues detected using bioinformatics methods.

Notes: (A) AVL9 expression in CRC tissues and normal tissues was analyzed using the GEPIA database. *P $<0.05$. (B) AVL9 expression was upregulated in CRC using the GSE32323 GEO dataset. (C) TCGA data also indicated that AVL9 expression was upregulated in CRC versus normal tissues.

Abbreviations: CRC, colorectal cancer; TCGA, The Cancer Genome Atlas. 

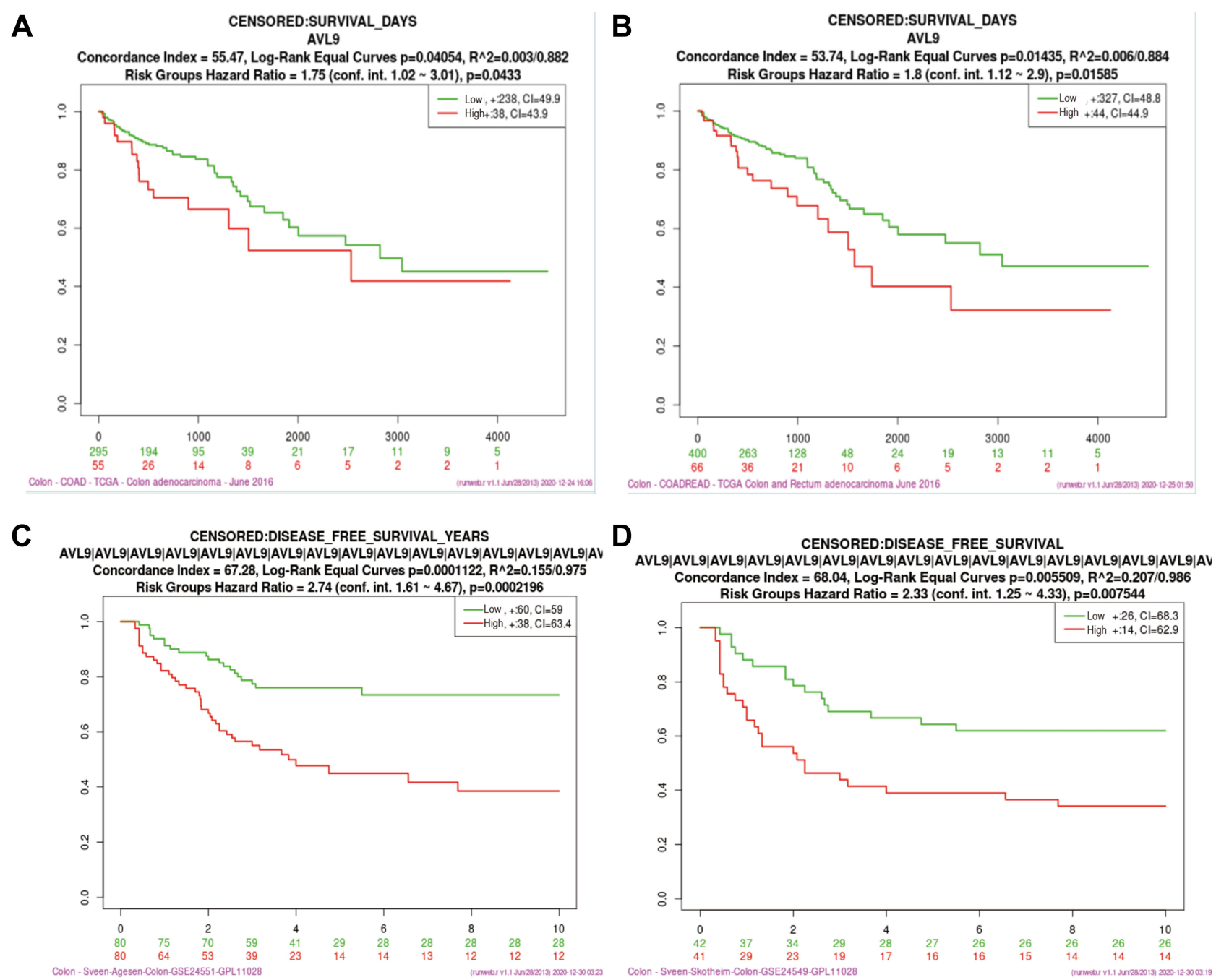

Figure 2 Analysis of the relationship between AVL9 expression and prognosis using bioinformatics methods.

Notes: (A and B) Kaplan-Meier survival analyses of OS using the TCGA data. (C and D) Kaplan-Meier survival analyses of DFS using GEO data.

Abbreviations: TCGA, The Cancer Genome Atlas; GEO, Gene Expression Omnibus; OS, overall survival; DFS, disease-free survival.

be/webtools/Venn/) to identify 160 genes that were common between the results of the two databases (Figure 3A), and GO function enrichment analysis of the DAVID6.8 database revealed that the relative expression genes were functionally concentrated in single organismal cell-cell adhesion, post-transcriptional regulation of gene expression, and negative regulation of vascular endothelial growth factor receptor signaling pathway (Figure 3B, $\mathrm{P}<$ 0.05). The KEGG pathway analysis showed that these genes were mainly involved in progesterone-mediated oocyte maturation, axon guidance, insulin signaling pathway, and ubiquitin-mediated proteolysis signaling pathways (Figure 3C, $\mathrm{P}<0.05$ ). PPI network analysis using Cytoscape 3.7.1 showed that the KBTBD2, KIAA1147, $R N F 216, E P D R 1$, and $A N K I B 1$ genes were most closely related to $A V L 9$ (Figure 4). Furthermore, according to the GEPIA data, we identified that the correlation between KBTBD2, KIAA1147, RNF216, EPDR1, and AVL9 were all positively correlated according to Pearson's correlation coefficient (Figure S3A-D, $\mathrm{P}=0$ ).

\section{AVL9 is Upregulated in Clinical Samples and Associated with the Clinical Characteristics of CRC}

To validate the relationship between $A V L 9$ expression and clinicopathological characteristics, we detected AVL9 in 50 paired CRC tissues and adjacent normal tissues. Reverse transcription-quantitative polymerase chain reaction (RTqPCR) showed that $A V L 9$ expression was higher in $\mathrm{CRC}$ 
A

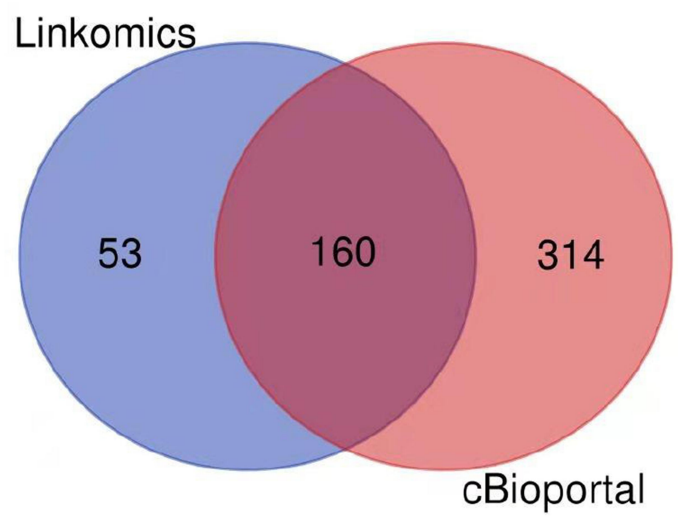

C

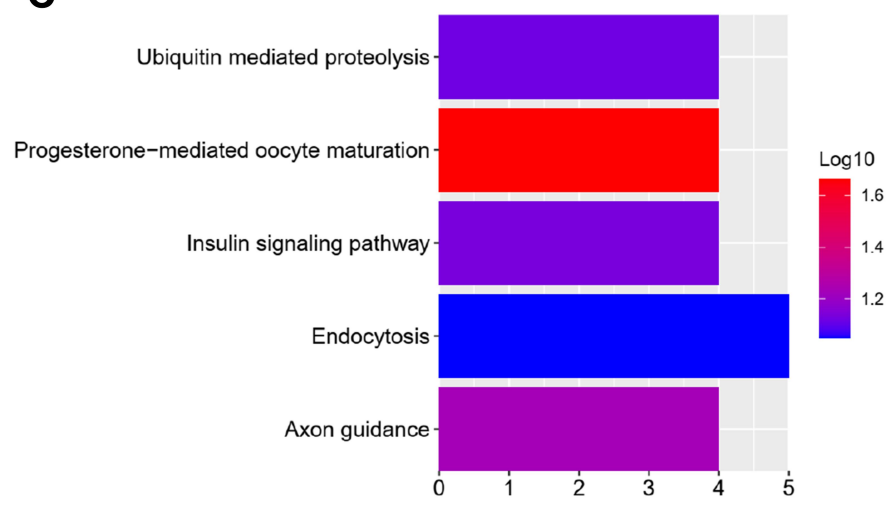

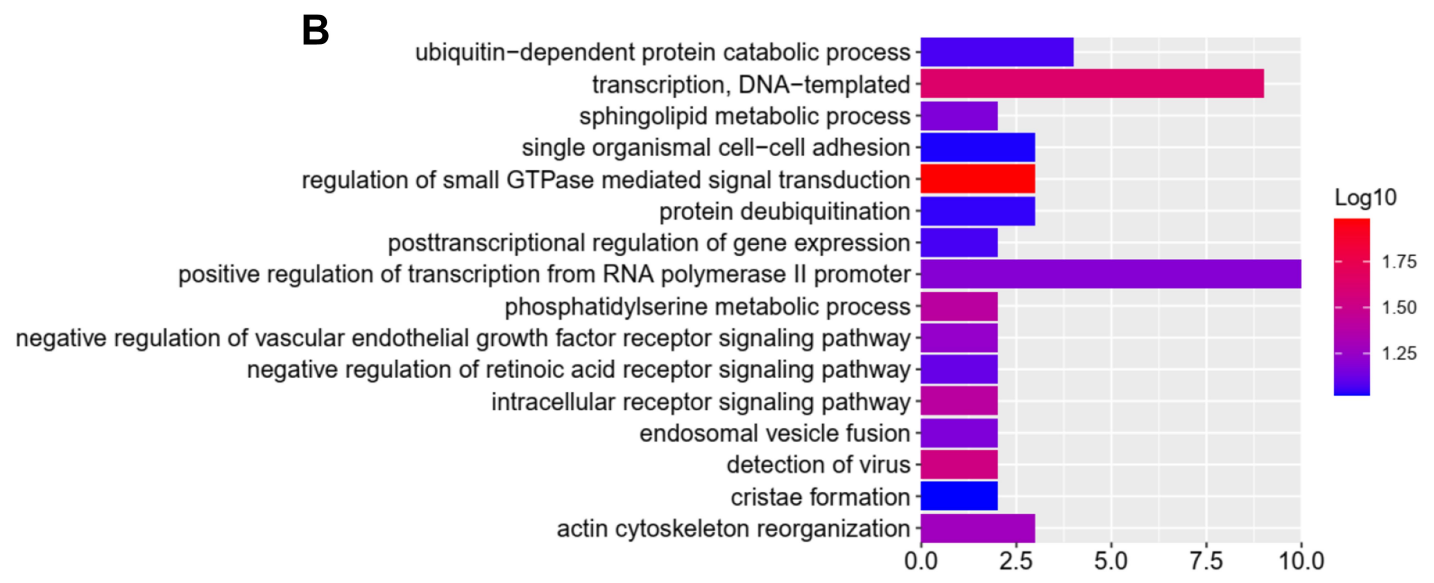

Figure 3 Bioinformatics analysis of genes related to AVL9 expression.

Notes: (A) A total of 160 AVL9 relative genes were common between the results of the LinkedOmics and cBioportal databases. (B) GO analysis of AVL9 expression-related genes. (C) KEGG analysis of AVL9 expression-related genes.

Abbreviations: GO, gene ontology; KEGG, Kyoto Encyclopedia of Genes and Genome.

tissues than in the adjacent normal tissues (Figure 5A, $\mathrm{P}<$ 0.001). Furthermore, patients with higher $A V L 9$ expression had poorer OS times (Figure 5B, $\mathrm{P}<0.0001$ ) than those with lower $A V L 9$ expression. In addition, $A V L 9$ was more likely to be highly expressed in patients with TNM stage III or IV than in patients with TNM stage I or II (Figure 5C, P < 0.0001). Compared to patients with well-to-moderate, $A V L 9$ expression in patients with poor-to-moderate was significantly increased (Figure $5 \mathrm{C}$ and $\mathrm{D}, \mathrm{P}<0.001$ ). Moreover, the ROC curve analysis was used to analyze the predictive value of $A V L 9$ expression in the tissues of patients with CRC. The cutoff value of 1.296 had a sensitivity of $72.9 \%$ and a specificity of $64 \%$, while the area under the curve (AUC) was 0.683 (Figure 5E). To analyze the relationship between $A V L 9$ expression and the clinical parameters of CRC patients, we divided them into high and low expression groups according to the medium expression value of $A V L 9$ as the cutoff point (Table 1). Our data confirmed that $A V L 9$ expression showed statistically significant differences according to differentiation ( $\mathrm{P}=0.01)$ and TNM stage $(\mathrm{P}=$ $0.028)$ but not according to sex $(\mathrm{P}=0.729)$, age $(\mathrm{P}=0.470)$, tumor size $(\mathrm{P}=0.064)$, or $\mathrm{N}$ status $(\mathrm{P}=0.204)$. Furthermore, multivariate survival analysis (Table 2) identified the AVL9 expression level as a covariate (hazard ratio [HR], 5.695; 95\% confidence interval [CI], 1.860-17.442, $\mathrm{P}=0.002$ ). Collectively, upregulated expression of $A V L 9$ may serve as a biomarker for CRC detection.

\section{AVL9 Was Upregulated in the Plasma of CRC Patients}

We next confirmed the expression of $A V L 9$ in 60 samples from CRC patient plasma and healthy control plasma based on the RT-qPCR assays, which confirmed that AVL9 expression in the plasma of CRC patients was remarkably upregulated versus that in the healthy controls 


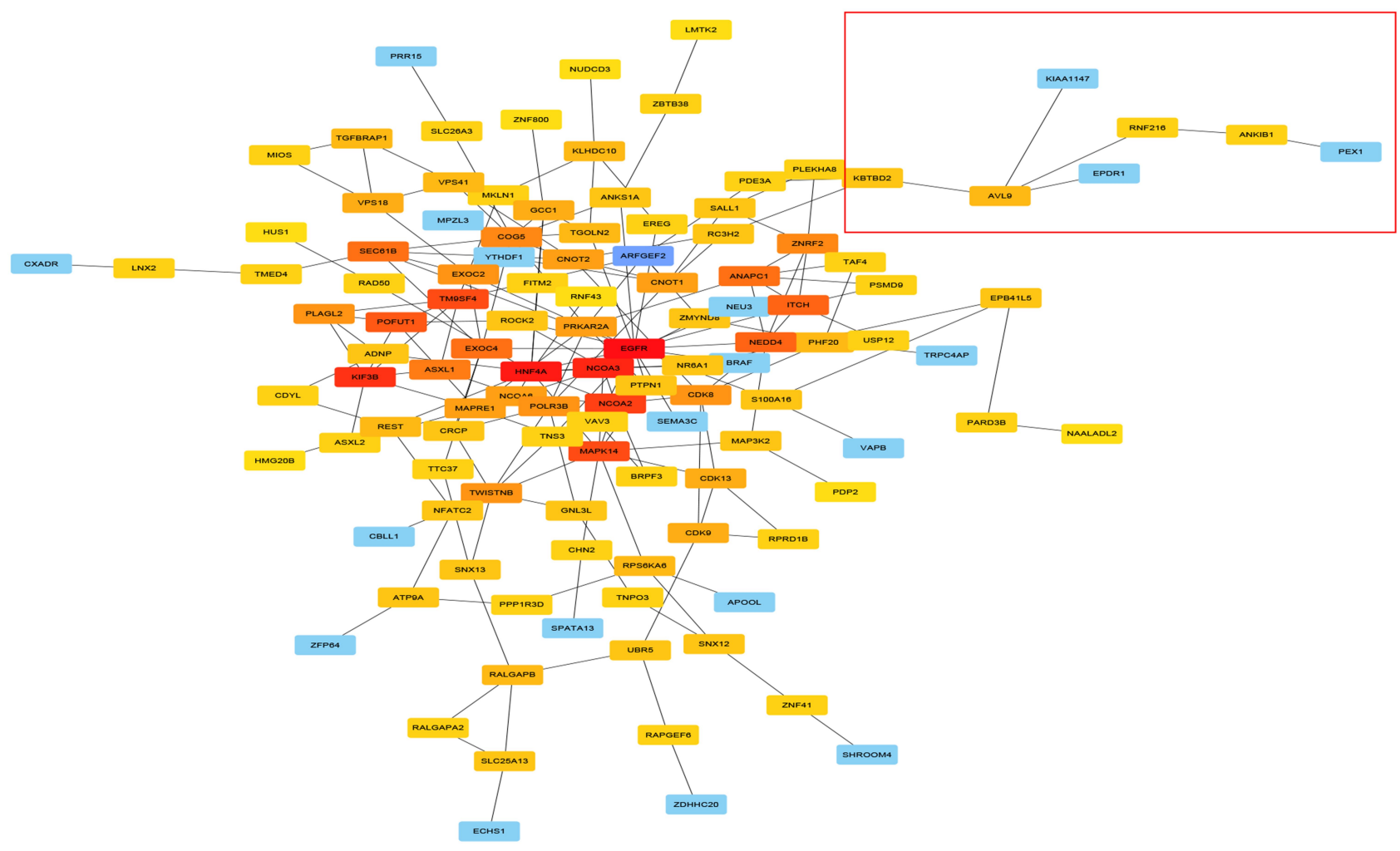

Figure 4 PPI network analysis of AVL9 expression-related genes and key gene predictions.

Notes: The nodes represent proteins, the edges represent the interaction of proteins, and the color depth of a node in the PPI network reflects interaction strength (order of interaction intensity: red $>$ deep yellow $>$ medium yellow $>$ light yellow $>$ purple $>$ blue).

Abbreviation: PPI, protein-protein interaction.

(Figure 6A, P $<0.01$ ). Subsequently, the ROC curve analysis was used to analyze the predictive value of $A V L 9$ in the plasma of patients with CRC. The cutoff value was 0.602 , with a sensitivity of $80.0 \%$ and specificity of $63.3 \%$, and the AUC was 0.729 (Figure 6B). Taken together, these results indicate that $A V L 9$ levels were elevated in $\mathrm{CRC}$ and might be useful as a non-invasive biomarker for detecting CRC in patients.

\section{Discussion}

CRC is one of the most common cancers and causes of cancer-related deaths worldwide. The high mortality rate of CRC patients is mainly due to the lack of biomarkers with high sensitivity and specificity. Many deaths can be avoided if CRC patients can be diagnosed and treated at an early stage. Therefore, determining effective biomarkers and prolonging the survival time of patients with CRC is of great clinical significance. ${ }^{9}$ AVL9, a migration-associated gene, was first described in budding yeast as an exocytosis gene, ${ }^{10}$ and recent studies have shown that it plays an important role in cell polarity, cell migration, and cell cycle progression., ${ }^{4,5}$ For instance, AVL9 expression is reportedly upregulated in clear cell renal carcinomas and promotes cell migration, ${ }^{6}$ and Liang et $\mathrm{al}^{11}$ revealed that $A V L 9$ is a direct target of miR-203a-3p, which promotes the proliferation, migration, and invasion of non-small cell lung cancer. In our previous study, we found that $A V L 9$ was a target gene of the linc00662/miR-497-5p axis, which promotes the occurrence and development of $\mathrm{CRC}{ }^{7}$ In present study, we aimed to increase our understanding of $A V L 9$ expression using bioinformatics analysis. The GEO and TCGA databases were used to identify that $A V L 9$ expression was upregulated in CRC tissues versus normal tissues, the survival time analysis showed that a higher level of $A V L 9$ led to worse OS and DFS, these results show that $A V L 9$ is a potential tumor-promoting gene of CRC.

To further study the mechanism of AVL9 expression upregulation in tumors, GO analyses showed that $A V L 9$, together with its related genes, was functionally concentrated in tumor-related functions, such as single organismal cell-cell adhesion, suggesting that they may be involved in the migration of immune cells and tumor cells ${ }^{12}$ and post-transcriptional regulation of gene expression and negative regulation of the vascular endothelial 
A

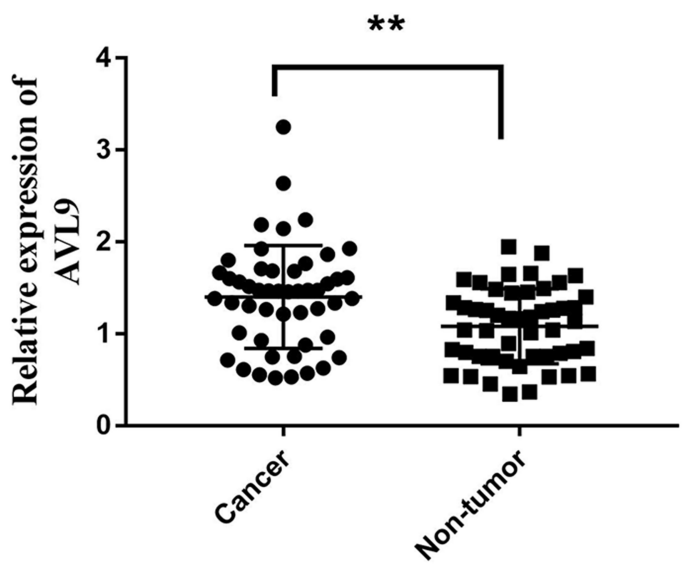

C

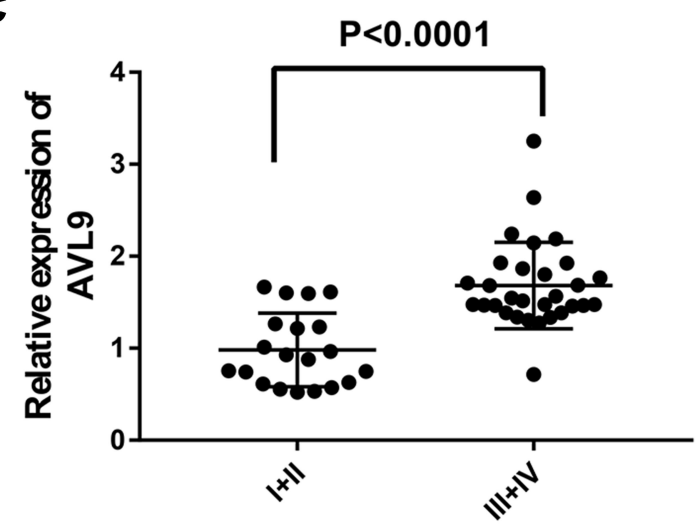

E

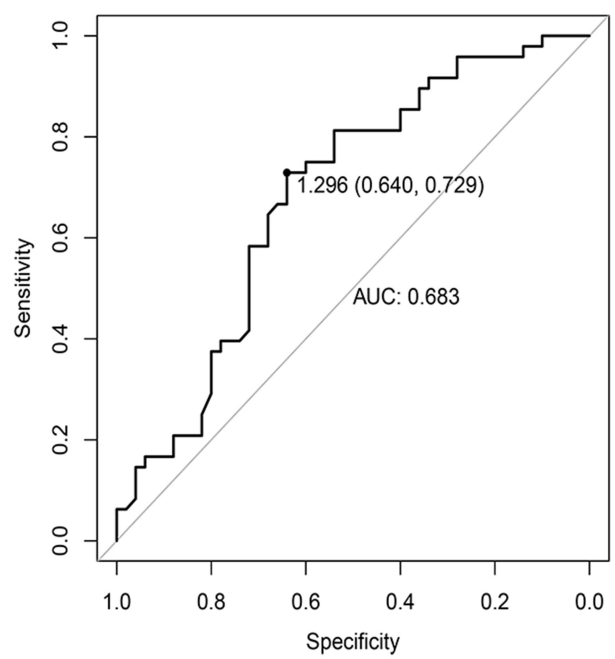

B

AVL9

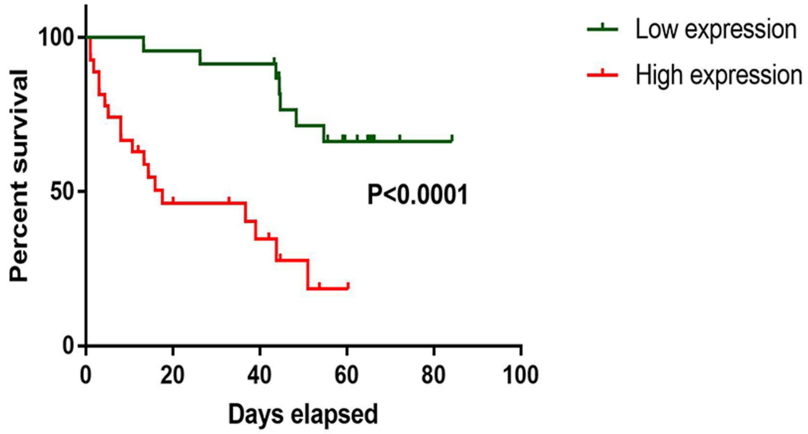

D

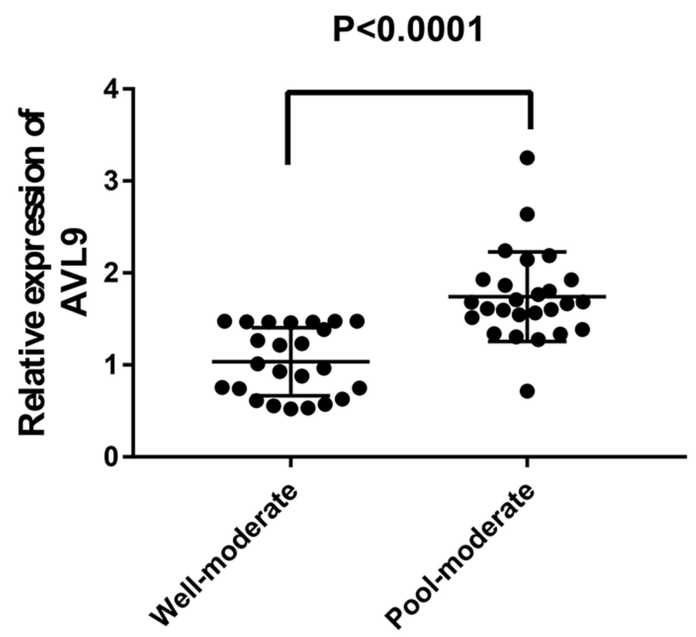

Figure 5 Expression level of AVL9 in CRC tissues.

Notes: (A) AVL9 was up-regulated in CRC tissues and analyzed by the reverse transcription-quantitative polymerase chain reaction assay. (B) Correlation between AVL9 expression and overall survival. (C) The expression of AVL9 was significantly higher in patients with TNM stage III or IV. (D) AVL9 expression in patients with poor to moderate tumor differentiation was increased. (E) The receiver operating characteristic curve for predicting CRC was analyzed using normal tissues as a control. The cutoff value was I.296, with a sensitivity of 0.729 and specificity of 0.640 , and the AUC was 0.6830 . $* * P<0.01$.

Abbreviations: CRC, colorectal cancer; AUC, area under the curve.

growth factor receptor signaling pathway, which shed light on the mechanism of cancer in cell growth, differentiation, development, and apoptosis. ${ }^{13}$
KEGG pathway analyses also revealed that these genes were mainly involved in signaling pathways that promote tumor development. For instance, it has been well known 
Table I AVL9 Expression and Clinicopathological Features in Patients with Colorectal Cancer

\begin{tabular}{|c|c|c|c|c|}
\hline \multirow[t]{2}{*}{ Feature } & \multirow[t]{2}{*}{$\mathbf{n}$} & \multicolumn{2}{|c|}{ AVL9 Relative Expression } & \multirow[t]{2}{*}{$P$ value* } \\
\hline & & High & Low & \\
\hline \multicolumn{5}{|l|}{ Gender } \\
\hline Male & 26 & 11 & 15 & 0.729 \\
\hline Female & 24 & 9 & 15 & \\
\hline \multicolumn{5}{|l|}{ Age } \\
\hline$\leq 50$ years & 18 & 6 & 12 & 0.470 \\
\hline$>50$ years & 32 & 14 & 18 & \\
\hline \multicolumn{5}{|l|}{ Tumor size } \\
\hline$\leq 5 \mathrm{~cm}$ & 27 & 14 & 13 & 0.064 \\
\hline$>5 \mathrm{~cm}$ & 23 & 6 & 17 & \\
\hline \multicolumn{5}{|l|}{ Differentiation } \\
\hline Well-moderate & 24 & 7 & 17 & 0.001 \\
\hline Poor & 26 & 20 & 6 & \\
\hline \multicolumn{5}{|l|}{$\mathrm{N}$ status } \\
\hline Negative & 23 & 10 & 13 & 0.203 \\
\hline Positive & 27 & 16 & 11 & \\
\hline \multicolumn{5}{|l|}{ TNM stage } \\
\hline I+II & 20 & 7 & 13 & 0.028 \\
\hline III+IV & 30 & 20 & 10 & \\
\hline
\end{tabular}

Notes: Statistical analysis was performed by Pearson $\chi^{2}$ test. *Value in bold indicates $P<0.05$.

that endocytosis plays a causative role in the cell homeostasis and proliferation control of cancer ${ }^{14}$ Similarly, previous studies reported that the insulin signaling pathway can promote tumor growth and invasion. ${ }^{15}$ Furthermore, it is well known that nerves and blood vessels have similar abilities to form branch networks, and there is strong evidence that the development of nerve and tumor vascular systems is regulated by axon-guided receptors and ligands. ${ }^{16}$ Ubiquitin-regulated protein degradation is necessary for useless proteins or proteins synthesized incorrectly in cells, and studies have shown that the dysregulation of ubiquitin activity can promote tumor progression. ${ }^{17}$ In summary, these findings suggest that $A V L 9$, as well as its related genes, affects tumor progression, invasion, and metastasis. In the present study, the PPI network showed that $A V L 9$ directly interacts with KBTBD2, KIAA1147, RNF216, and EPDR1; a further study found that all were positively correlated with $A V L 9$ according to the Pearson's correlation coefficient, and recent studies demonstrated that KBTBD2, KIAA1147, $R N F 216$, and EPDR1 play an important role in the tumorigenesis and prediction of progression in cancer, ${ }^{18-21}$ that is, these genes may collaborate to promote the progression of $\mathrm{CRC}$, but the specific mechanism among them requires further analysis in future studies.

To further verify the expression of $A V L 9$ in CRC tissues and adjacent normal colorectal mucosa using RT-qPCR, we revealed that $A V L 9$ expression was significantly increased in CRC tissues and closely associated with TNM staging and degree of differentiation. Furthermore, our data also indicated that the $A V L 9$ low-expression group had a longer survival time than the high-expression group. In CRC diagnosis, owing to advantages such as convenience, minimally invasive, cheap, and repeatability, liquid biopsy provides a vital function versus colonoscopy examinations; however, the most commonly used diagnostic markers, namely, carcinoembryonic antigen, carbohydrate antigen19-9, and carbohydrate antigen $72-4$, exhibit low specificity and sensitivity, particularly in early-stage CRC. ${ }^{22}$ Therefore, the main priority is to identify novel biomarkers to reliably detect early CRC and relapse in patients post-surgery, and emerging research has demonstrated that circulating tumor cells, ctDNA, miRNAs, and lncRNAs can improve CRC screening and diagnosis and predict recurrence and metastasis. ${ }^{23}$ In this study, to explore the potential diagnostic role of $A V L 9$ in

Table 2 Univariate and Multivariate Analyses of Clinical Pathological Factors of OS in 50 Patients with CRC

\begin{tabular}{|c|c|c|c|c|c|c|c|c|}
\hline \multirow{3}{*}{$\begin{array}{l}\text { Clinical Parameters } \\
\text { Gender (male vs female) }\end{array}$} & \multicolumn{4}{|c|}{ Univariate } & \multicolumn{4}{|c|}{ Multivariate } \\
\hline & \multirow{2}{*}{$\begin{array}{c}\text { HR } \\
1.023\end{array}$} & \multicolumn{2}{|c|}{$95 \% \mathrm{Cl}$} & \multirow{2}{*}{$\begin{array}{c}\mathbf{P} \\
0.954\end{array}$} & \multirow{2}{*}{$\begin{array}{c}\text { HR } \\
1.209\end{array}$} & \multicolumn{2}{|c|}{$95 \% \mathrm{Cl}$} & \multirow{2}{*}{$\begin{array}{c}P \\
0.661\end{array}$} \\
\hline & & 0.467 & 2.243 & & & 0.571 & 2.826 & \\
\hline Age $(<50$ vs $\geq 50)$ & 2.250 & 0.891 & 5.683 & 0.086 & 2.682 & 0.960 & 7.489 & 0.060 \\
\hline Differentiation (Well + moderate vs Poor + undifferentiated) & 2.167 & 0.965 & 4.868 & 0.061 & 0.793 & 0.260 & 2.414 & 0.683 \\
\hline Tumor size $(\mathbf{c m})(<5.0$ vs $\geq 5.0)$ & 0.759 & 0.343 & 1.678 & 0.496 & 0.347 & 0.130 & 1.104 & 0.075 \\
\hline TNM stage $(I+||$ vs III+IV) & 2.002 & 0.857 & 4.677 & 0.109 & 1.577 & 0.354 & 7.023 & 0.550 \\
\hline Lymphatic metastasis (Negative vs positive) & 1.557 & 0.968 & 3.472 & 0.280 & 1.322 & 0.349 & 5.006 & 0.861 \\
\hline AVL9 expression (Low vs High) & 5.522 & 2.197 & 13.87 & 0.000 & 5.695 & 1.860 & 17.442 & 0.002 \\
\hline
\end{tabular}

Note: Bold text in the table refer to statistically significant results. 
A

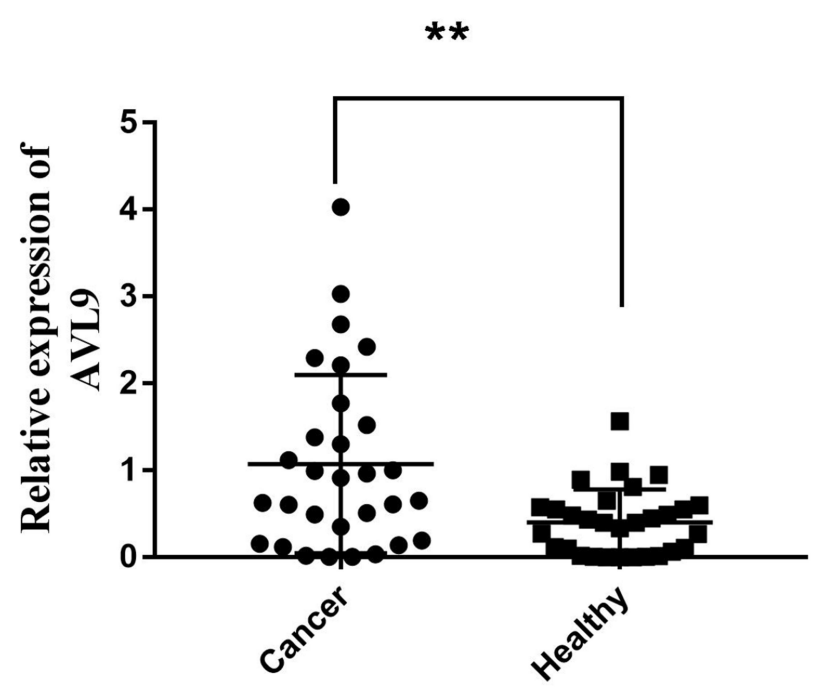

B

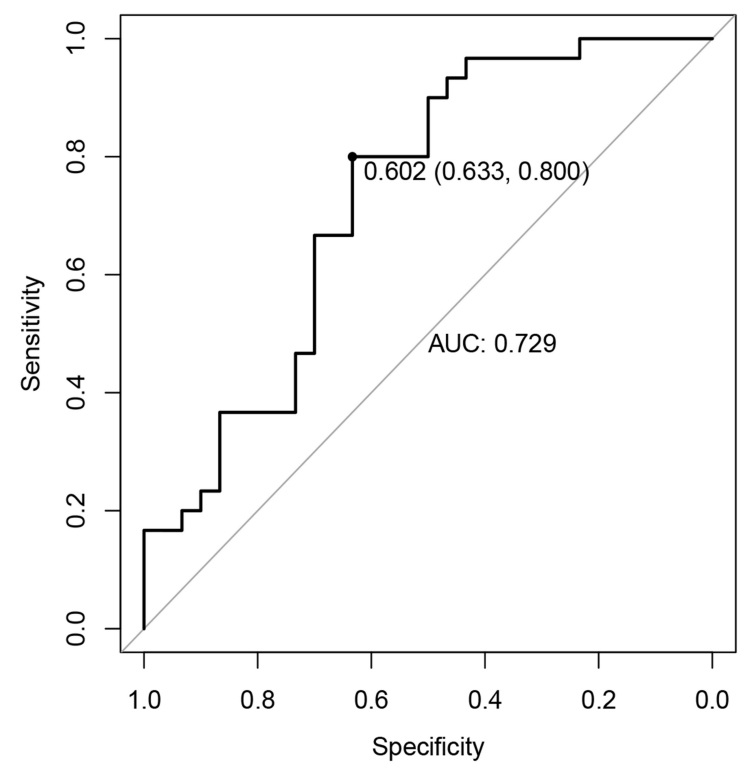

Figure 6 Diagnostic utility of circulating AVL9 was tested in 30 CRC patients and 30 healthy subjects.

Notes: (A) Expression levels of circulating AVL9 were upregulated in CRC patients versus healthy controls. (B) The AUC was 0.729 . The cutoff value was 0.602, with a sensitivity of 0.800 and a specificity of 0.633 . $* * P<0.01$.

Abbreviations: CRC, colorectal cancer; AUC, area under the curve.

$\mathrm{CRC}$, we first detected its expression in the plasma and found that it was significantly increased in CRC patients versus healthy controls; ROC curve analysis demonstrated $A V L 9$ detection in the tissues and plasma exhibited good sensitivity and specificity as a diagnostic marker for CRC, as the AUC of $A V L 9$ expression in the plasma was 0.729 with a cutoff value of 0.602 . However, due to the small number of patients, this study can explain only the detection of $A V L 9$, which plays a role in the early diagnosis of CRC, more large-scale studies and multicenter trials are still needed to confirm its advantages. Finally, AVL9 is expected to become a new biomarker of liquid biopsy for enabling the early diagnosis of CRC; assessment of progress, treatment effects, and prognosis as well as the evaluation of chemotherapy-acquired drug resistance in the near future.

\section{Conclusion}

We demonstrated here that the high expression and overexpression of $A V L 9$ in CRC indicate poor prognosis. Detection of $A V L 9$ in the tissues and plasma showed good efficacy, which revealed that it could act as a diagnostic marker for CRC.

\section{Acknowledgments}

This work was financially supported by the Shantou Science and Technology Bureau (No. 2006241552607),
"Dengfeng Project" for the construction of high-level hospitals in Guangdong Province -the First Affiliated Hospital of Shantou University Medical College Supporting Funding (NO.202003-17).

\section{Disclosure}

The authors declare no conflicts of interest.

\section{References}

1. Cheng L, Eng C, Nieman LZ, et al. Trends in colorectal cancer incidence by anatomic site and disease stage in the United States from 1976 to 2005. Am J Clin Oncol. 2011;34(6):573-580. doi:10.1097/COC.0b013e3181fe41ed

2. Smith RA, von Eschenbach AC, Wender R, et al. American Cancer Society guidelines for the early detection of cancer: update of early detection guidelines for prostate, colorectal, and endometrial cancers. Also: update 2001-testing for early lung cancer detection. CA Cancer J Clin. 2001;51(1):38-75. quiz 77-80. doi:10.3322/canjclin.51.1.38

3. Siegel RL, Miller KD, Goding Sauer A, et al. Colorectal cancer statistics, 2020. CA Cancer J Clin. 2020;70(3):145-164. doi:10.3322/caac.21601

4. Linford A, Yoshimura S, Nunes Bastos R, et al. Rab14 and its exchange factor FAM116 link endocytic recycling and adherens junction stability in migrating cells. Dev Cell. 2012;22(5):952-966. doi:10.1016/j.devcel.2012.04.010

5. Li Y, Xu J, Xiong $\mathrm{H}$, et al. Cancer driver candidate genes AVL9, DENND5A and NUPL1 contribute to MDCK cystogenesis. Oncoscience. 2014;1(12):854-865. doi:10.1016/j.devcel.2012.04.010

6. Zhang W, Wang J, Chai R, et al. Hypoxia-regulated lncRNA CRPAT4 promotes cell migration via regulating AVL9 in clear cell renal cell carcinomas. Onco Targets Ther. 2018;11:4537-4545. doi:10.2147/ OTT.S169155 
7. Wang $\mathrm{H}, \mathrm{Yu} \mathrm{M}, \mathrm{Hu} \mathrm{W}$, et al. Linc00662 promotes tumorigenesis and progression by regulating miR-497-5p/AVL9 axis in colorectal cancer. Front Genet. 2020;10:1385. doi:10.3389/fgene.2019.01385

8. Robin X, Turck N, Hainard A, et al. pROC: an open-source package for $\mathrm{R}$ and $\mathrm{S}+$ to analyze and compare ROC curves. BMC Bioinform. 2011;12(1):77. doi:10.1186/1471-2105-12-77

9. Siegel R, DeSantis C, Virgo K, et al. Cancer treatment and survivorship statistics, 2012. CA Cancer J Clin. 2012;62:220-241. doi:10.3322/caac. 21149

10. Harsay E, Schekman R. Avl9p, a member of a novel protein superfamily, functions in the late secretory pathway. Mol Biol Cell. 2007;18(4):1203-1219. doi:10.1091/mbc.e06-11-1035

11. Liang J, Sun T, Wang G, et al. Clinical significance and functions of miR-203a-3p/AVL9 axis in human non-small-cell lung cancer. Per Med. 2020;17(4):271-282. doi:10.2217/pme-2019-0108

12. Friedl P, Gilmour D. Collective cell migration in morphogenesis, regeneration and cancer. Nat Rev Mol Cell Biol. 2009;10 (7):445-457. doi:10.1038/nrm2720

13. Corbett AH. Post-transcriptional regulation of gene expression and human disease. Curr Opin Cell Biol. 2018;52:96-104. doi:10.1016/j. ceb.2018.02.011

14. Polo S, Pece S, Di Fiore PP. Endocytosis and cancer. Curr Opin Cell Biol. 2004;16(2):156-161. doi:10.1016/j.ceb.2004.02.003

15. Varewijck AJ, Janssen JA. Insulin and its analogues and their affinities for the IGF1 receptor. Endocr RElat Cancer. 2012;19(5):F63F75. doi:10.1530/ERC-12-0026

16. Klagsbrun M, Eichmann A. A role for axon guidance receptors and ligands in blood vessel development and tumor angiogenesis. Cytokine Growth Factor Rev. 2005;16(4-5):535-548. doi:10.1016/j. cytogfr.2005.05.002
17. Khan OM, Carvalho J, Spencer-Dene B, et al. The deubiquitinase USP9X regulates FBW7 stability and suppresses colorectal cancer. J Clin Invest. 2018;128(4):1326-1337. doi:10.1172/JCI97325

18. Dai K, Huang Y, Chen Z, Sun X, Yang L, Jiang Y. Kbtbd2 inhibits the cytotoxic activity of immortalized NK cells through down-regulating mTOR signaling in a mouse hepatocellular carcinoma model. Eur J Immunol. 2018;48(4):683-695. doi:10.1002/ eji.201747281

19. Taskesen E, Mishra A, van der Sluis S, et al; International FTDGenomics Consortium. Susceptible genes and disease mechanisms identified in frontotemporal dementia and frontotemporal dementia with amyotrophic lateral sclerosis by DNA-methylation and GWAS. Sci Rep. 2017;7(1):8899. doi:10.1038/s41598-017-09320-z

20. Wang H, Wang Y, Qian L, et al. RNF216 contributes to proliferation and migration of colorectal cancer via suppressing BECN1-dependent autophagy. Oncotarget. 2016;7 (32):51174-51183. doi:10.18632/oncotarget.9433

21. Gimeno-Valiente F, Riffo-Campos ÁL, Ayala G, et al. EPDR1 up-regulation in human colorectal cancer is related to staging and favours cell proliferation and invasiveness. Sci Rep. 2020;10(1):3723. doi:10.1038/s41598-020-60476-7

22. Carpelan-Holmström M, Louhimo J, Stenman UH, et al. CEA, CA 19-9 and CA 72-4 improve the diagnostic accuracy in gastrointestinal cancers. Anticancer Res. 2002;22(4):2311-2316.

23. Vacante M, Ciuni R, Basile F, Biondi A. The liquid biopsy in the management of colorectal cancer: an overview. Biomedicines. 2020;8 (9):308. doi:10.1159/000494411

\section{Publish your work in this journal}

Cancer Management and Research is an international, peer-reviewed open access journal focusing on cancer research and the optimal use of preventative and integrated treatment interventions to achieve improved outcomes, enhanced survival and quality of life for the cancer patient.
The manuscript management system is completely online and includes a very quick and fair peer-review system, which is all easy to use. Visit http://www.dovepress.com/testimonials.php to read real quotes from published authors. 